\title{
A Quantum Confinement Study of the Electronic Energy of Some Nanocrystalline Silicon Quantum-Dots
}

\author{
Sylvester A. Ekong ${ }^{1 *}$ and Mike. O. Osiele ${ }^{2}$ \\ ${ }^{1)}$ Department of Physics and Energy Studies, Achievers University, Owo, Nigeria \\ 2) Department of Physics, Delta State University, Abraka, Nigeria \\ Corresponding author: Email: ani_sly@yahoo.co.uk
}

Keywords: Quantum confinement, Shape, Quantum-dot, nc-Si, IQW.

\begin{abstract}
We have employed a quantum confinement (QC) model to the study of different shapes of nanocrystalline silicon (nc-Si) quantum dot. Each dots (shapes), although within the limits of an effective diameter of $3 \mathrm{~nm}$, exhibits divergence leading to different electronic energy based on the transitions from the quantum selection rule. Also, the graphical representation of the energies from each shape as a function of the effective diameter gives a qualitatively similar spectrum of discrete energies. The results obtained in this work using QC model are in good agreement with experiment and other models in literature.
\end{abstract}

\section{INTRODUCTION}

With the recent progress in the last years of novel technologies in semiconductor growth, the discoveries in the physics of low-dimensional systems such as nanocrystals have concurrently opened new channels in this extremely active field of both theoretical and experimental research [1, 2]. The nanocrystalline silicon (nc-Si) is extremely investigated, not only because of its various applications, but also because of the interest presented by new phenomena that takes place at nanometric scale. Among the new nanoscale [3-6] phenomena, a leading place belongs to the quantum confinement (QC) [3, 4]. Quantum confinement in nano semiconductors (which occurs when electrons are strongly confined in one, two or three dimensions), is the basis behind research and development of low-dimensional semiconductor nanostructures [7]. QC effect plays a major role in the behaviour of the nanocrystal systems. In the case of the nanocrystalline silicon (nc-Si), quantum confinement is dominant in transport processes and has an important contribution in their electronic phenomena. Silicon nanocrystalline properties which are derived from this effect, are very sensitive to shape and size $[3,5,6,8]$ distributions within the sample. It is therefore important to control this size with a maximum accuracy. Nanocrystalline silicon (nc-Si) is obviously compatible with the classical silicon-based devices [9-12]. The quantum confinement determines the enlargement of the band gap [13] and leads to the breakdown of the momentum conservation rule [14], as well as the appearance of new energy levels and supplementary transitions [11, 12]. The calculation of the binding and transition energies for a shallow donor impurity in cylindrical QWWs as a function of the wire radius have been reported [15]. In this paper, we have employed quantum confinement (QC) to study different shapes of nanocrystalline silicon (nc-Si) quantumdots based on the quantum selection rule and investigating their size dependent electronic energy as a function of their effective diameter on single material level in order to reveal the basic mechanism of its electronic properties as the main goals of the present work. The rest of this paper is organized as follows: Section 2 presents the theoretical formalism, and in Section 3 the results and discussion are presented. Finally, we draw a brief conclusion in Section 4.

\section{THEORETICAL FORMALISM}

Considering a nanocrystalline silicon material under a good first approximation where the electron Hamiltonian is given by the sum of a 1D Bloch-like longitudinal part and a 2D infinite quantum well (IQW) transversal part, the electronic energy generated from quantum confinement (QC) model is given as: $[4,12]$ 


$$
E=\varepsilon_{n, k_{z}}+\frac{2 \pi^{2} \hbar^{2}}{m^{*} d^{2}} X_{l, p}^{2}
$$

which can be written as

$$
E=\varepsilon_{n, k_{z}}^{s}+E_{l, p-1}
$$

where $E_{l, p-1}$ is the discrete energy level for transversal motion, and $\varepsilon_{n, k_{z}}^{s}$ is the shifted longitudinal Bloch energy in order to have $E_{0,0} \equiv 0$. Since the energy is spin, s, independent, leading to a disappearance of the first term, equation (2) is then reduced to

$$
E_{l, p}=\frac{2 \pi^{2} \hbar^{2}}{m^{*} d^{2}}\left(X_{l, p+1}^{2}-X_{0,1}^{2}\right)
$$

where $m^{*}=0.66 m_{e}$ is the transversal effective electron mass, $d$ is the effective diameter and the terms in bracket are transitions between quantum confinement levels conditioned by the quantum selection rules $[4,9]$. For a cylindrical shape, $z_{l, p}=\pi x_{l, p}$ is the $p$-th zero of the Bessel function $J_{l}(z)$, [16] and $l$ being the orbital quantum number. In the following, the QC levels will be quoted $(l, p)$. For a square prism, equation (3) becomes

$$
E_{l, p}=\frac{2 \pi^{2} \hbar^{2}}{m^{*} d^{2}}\left(X_{l, p+2}^{2}-X_{0,2}^{2}\right)
$$

where

$$
\begin{aligned}
& X_{l, p+2}=(1 / 2) \sqrt{l^{2}+(p+2)^{2}}, \\
& p+2=n_{x}+n_{y}, \\
& l=\left|n_{x}-n_{y}\right|
\end{aligned}
$$

where $n_{x}$ and $n_{y}$ are the corresponding quantum numbers for a two dimensional quantum well, and $l=0$ means that there is no orbital motion.

Quantum dots are spherical in shape. The quantum confinement energy levels are

$$
E_{l, p}=\frac{2 \pi^{2} \hbar^{2}}{m^{*} d^{2}}\left(X_{l, p+1}^{2}-X_{0,1}^{2}\right)
$$

where $z_{l, p}=\pi x_{l, p}$ is the $p$-th zero of the spherical Bessel function. Also, $l$ is the orbital quantum number, and for a spherical quantum dot $m^{*}=m_{e}$.

\section{RESULTS AND DISCUSSION}

We have considered a cylindrical, square, and spherical shapes of quantum-dots made of nanocrystalline silicon (nc-Si) in this work with the results as presented in Figures 1, 2 and 3. The Figures 1, 2 and 3 shows a graphical representation of the electronic energy as a function of the effective diameter of various shapes with regards to different quantum numbers $(1,1 ; 1,2 ; 1,3 ; 1,4$; and 1,5 ) in the absence of the longitudinal part. For a very small dot effective diameter (i.e. $d<$ $2 \mathrm{~nm}$, strong radial confinement), the electronic energy diverges as the effective diameter (d) tends to zero (i.e. $\mathrm{d} \rightarrow 0$ ) in a several fashion for different quantum numbers. The divergence in each case for a strong axial confinement $\left(z_{l, p}<2\right)$ is more vivid than in the weak axial case $\left(z_{l, p}>2\right)$. The electron is squeezed in smaller diameter than in latter cases. From the graphs, the weak radial confinement $(\mathrm{d} \geq 3 \mathrm{~nm})$ and strong axial confinement case indicate that the electronic energy tends to the value of the two dimensional hydrogen donor $\left(\mathrm{E}_{\mathrm{b}} \rightarrow 4 \mathrm{~d}\right)[17]$. 
In the case of finite height potential barrier, the electronic energy increases with decreasing effective diameter of the nanocrystalline silicon dot, reaching a maximum value and finally decreases exponentially with increasing effective diameter of the dots. Again, while the nc-Si effective diameters becomes very large $(\mathrm{d}>>3 \mathrm{~nm})$, the ground state electronic energy converges to the corresponding bulk values because the electrons no longer interacts with the nc-Si QD boundaries, hence any impurity introduced will behave like a free hydrogen atom.

Also, for effective diameters greater than $3 \mathrm{~nm}$, there are no more energy bands but permissible quasi-bands formed by neighboring QC energy levels, separated by relatively large intervals known as forbidden bands. Due to the small number of atoms in a nc-Si dots, the forbidden bands are increased

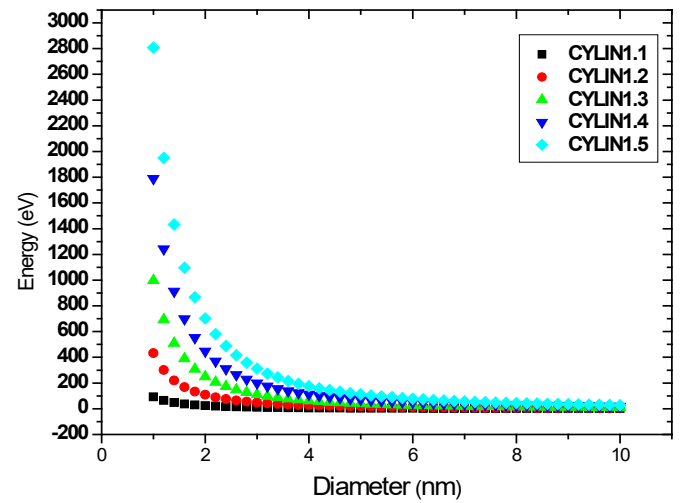

Figure 1: The electronic energy as a function of effective diameter for cylindrical shape with different quantum numbers.

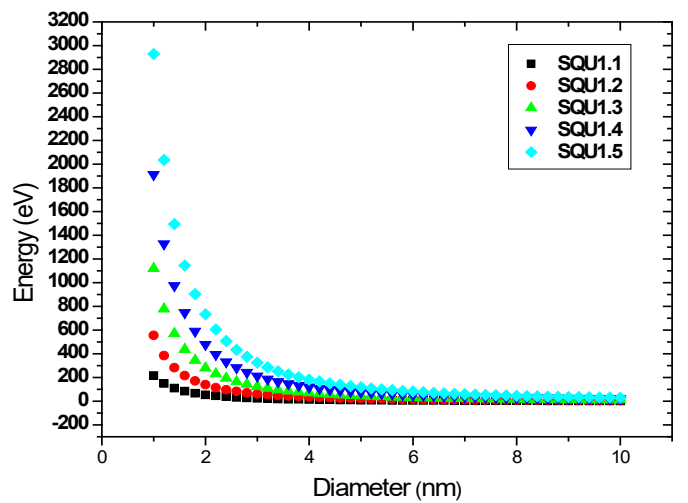

Figure 2: The electronic energy as a function of effective diameter for Square shape with different quantum numbers.

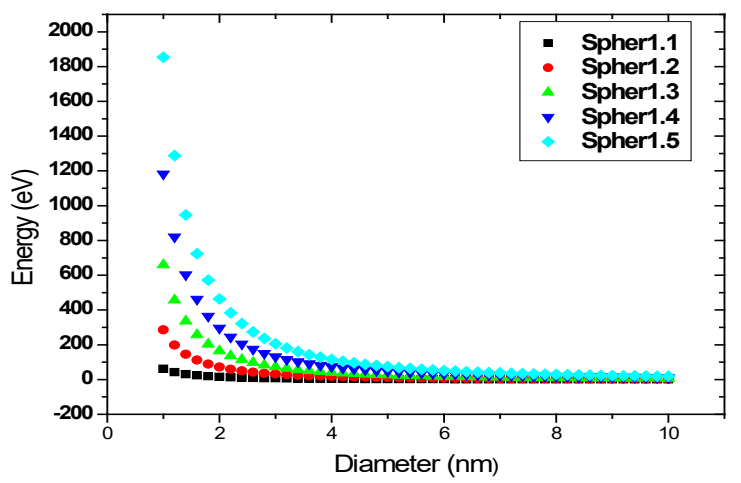

Figure 3: The electronic energy as a function of effective diameter for Spherical shape with different quantum numbers.

with respect to the bulk crystal materials (with more than $100 \%$ for sizes of the order of $3 \mathrm{~nm}$ ) tending to a direct band. This indicates that the momentum conservation law no longer holds, due to the reduction in number of states in a quasi-band [18]. In the Figures, the QC energy level for which $n_{x}=1$ and $n_{y}=5$ (i.e. CYLIN 1,5; SQU 1,5 and Spher 1,5) has the highest energy while the QC level for which $n_{x}=1$ and $n_{y}=1$ (i.e. CYLIN 1,1; SQU 1,1 and Spher 1,1) has the least energy, followed by the level for which $n_{x}=1$ and $n_{y}=2$ (i.e. CYLIN 1,2; SQU 1,2 and Spher 1,2). This shows that the energy of a particular level depends on the difference between the quantum numbers at that level. Nevertheless, Figures 1, 2 and 3 have a transition in their QC energy levels leading to a bunch as their quantum numbers increases higher. Another close view on the graphs of the nc-Si quantum dots shows that the increase in the electronic energy will be more pronounced for large dot sizes. However, the results are in perfect conformity with other experimental predictions [3, 4, 19], 
which states that, all three shapes will have quantitatively the same results; and that the confinement of electrons in various dimensions always lead to a qualitatively similar spectrum of discrete energies. This is true for a broad class of potential wells, irrespective of their dimensionality and shape. Thus, from the Figures, it is vividly observed that the energy of the nanocrystalline silicon material is maximum in square shapes, followed by cylindrical shapes and least in spherical shapes. A possible reason for this may be due to discretization of the QC energy levels as a result of changes in dimensions [19].

\section{CONCLUSION}

Quantum confinement (QC) study on nanocrystalline silicon (nc-Si) quantum dots have been carried out to study dominant role played on the electronic properties of the different shapes of the nc-Si quantum dot. The model allows for the identification of the energy transitions between the different shapes based on the quantum selection rules. The confinement of electrons in various dimensions leads to a qualitatively similar spectrum of energy discretization. However, the computed results obtained are in good conformity with other experimental predictions and models in literature.

\section{References}

[1] Mmadi, A., I. Zorkani, K. Rahmani and A. Jorio, (2013), Magnetic field effect on the diamagnetic susceptibility of hydrogenic donor in cylindrical quantum dot, The African Review of Physics, 8:0033, pp. 219.

[2] Räsänen, E., H. Saarikoski, V. N. Stavrou, A. Harju, M. J. Puska, and R. M. Nieminen, (2008): Electronic structure of rectangular quantum dots, pp. 1. [Unpublished]

[3] Chih-Hsien Cheng, Yu-Chung Lien, Chung-Lun Wu, and Gong-Ru Lin, (2013): Multicolor electroluminescent $\mathrm{Si}$ quantum dots embedded in $\mathrm{SiO}_{\mathrm{X}}$ thin film MOSLED with $2.4 \%$ external quantum efficiency, Optics Express, Vol. 21, Issue 1, pp. 391-403.

[4] Ilya Sychugov, (2006): Synthesis properties of single luminescent silicon quantum dots, Royal Institute of Technology, Doctoral Thesis, Stockholm, Sweden, pp. 4-13, 32.

[5] Florian Maier-Flaig, Julia Rinck, Moritz Stephan, Tobias Bocksrocker, Michael Bruns, Christian Kübel, Annie K. Powell, Geoffrey A. Ozin and Uli Lemmer, (2013): Multicolor Silicon Light-Emitting Diodes (SiLEDs), American Chemical Society, Nano-letters, 13 (2), pp. 475-480.

[6] Chemical \& Engineering News, (2013): LEDs Made From Silicon Quantum Dots Shine in New Colors, Volume 91, Issue 5, pp. 8.

[7] Ali M. Mousa, and Karem H. Jawad, (2015), Performance of a Nano PbS/Si Hetrojunction Deposited by chemical spray pyrolysis, International Letters of Chemistry, Physics and Astronomy Vol. 51, pp. 13-16.

[8] Yang C. H., Rossi A., Ruskov R., Lai N. S., Mohiyaddin F. A., Lee S., Tahan C., Klimeck G., Morello A., and Dzurak A. S., (2013): Spin-valley lifetimes in a silicon quantum dot with tunable valley splitting, http://arxiv.org/pdf/1302.0983v1.pdf, [Cond-mat.mes-hall], pp. $1-11$.

[9] Ciurea M. L. and Iancu V., (2007): Why the Energy Levels Observed in Electrical Transport, Phototransport and Photoluminescence are Different, Academic Publications, Semiconductor, International Conference, Institute of Electrical and Electronics Enguneers, Volume 1, pp. 41-43. 
[10] Ioannou-Sougleridis V., Ouisse T., Nassiopoulou A. G., Bassani F. and Arnaud d'Avitaya F., (2001): Nonlinear electrical transport in $\mathrm{nc}-\mathrm{Si} / \mathrm{CaF}_{2}$ multilayer structures with ultrathin $\mathrm{CaF}_{2}$ layers, Journal of Applied Physics, Vol. 89, No 1, pp. 610.

[11] Iancu V., Stavarache I., and Ciurea M. L., (2006): Quantum confinement Modeling of Electrical and Optical Processes in nanocrystalline silicon, Journal of Optoelectronics and Advanced Materials Vol. 8, No 6, pp. 2156-2157.

[12] Ciurea M. L., (2005): Quantum confinement in nanocrystalline silicon, Journal of Optoelectronics and Advanced Materials Vol. 7, No 5, pp.2341, 2342 and 2344.

[13] Delerue C., Allan G., and Lannoo M., (1993): Phys. Rev. B 48, pp.11024.

[14] Heitmann J., Müller F., Yi L. X., Zacharias M., Kovalev D. and Eichhorn F., (2004), Phys. Rev. B 69, pp.195309.

[15] Villamila, P., C. Cabraa and N. P.Montenegro, (2005), Microelectronics J. 36, pp. 383.

[16] Abramovitz M and Stegun I. A, (1972): Handbook of Mathematical Functions with Formulas, Graphs, and Mathematical Tables, $10^{\text {th }}$ edition; National Bureau of Standards Applied Mathematics Series 55; Library of Congress Catalog Card Number: 64-60036; pp. $390,457$.

[17] Legoff, S. and B. Stébé, (1992), J. Phys. B 25, pp. 5261.

[18] Hu, G. Y., R. F. O’Connell, Y. L. He, and M. B. Yu, (1995), J. Appl. Phys. 78, pp. 3945.

[19] Poole Jr. C. P. and Owens F. A., (2003): Introduction to Nanotechnology, John Wiley and Sons, Inc. [ISBN 0-471-07935-9], pp. 226, 231-234. 